\title{
Temperature Resistance of Magnetoelastic Characteristics of 13CrMo4-5 Constructional Steel
}

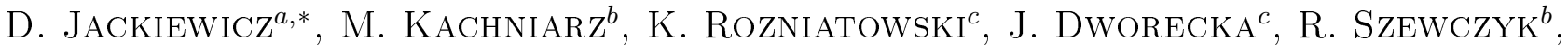 \\ J. SALACH ${ }^{b}$, A. BIENKOWSKI ${ }^{b}$ AND W. WiniaRSKI ${ }^{a}$ \\ ${ }^{a}$ Industrial Research Institute for Automation and Measurements PIAP, \\ Al. Jerozolimskie 202, 02-486 Warszawa, Poland \\ ${ }^{b}$ Institute of Metrology and Biomedical Engineering, Warsaw University of Technology, \\ A. Boboli 8, 02-525 Warszawa, Poland \\ ${ }^{c}$ Faculty of Materials Science and Engineering, Warsaw University of Technology, \\ Wołoska 141, 02-507 Warszawa, Poland
}

\begin{abstract}
In the paper the results of investigation on tensile stresses dependence of magnetoelastic characteristics of cores made of $13 \mathrm{CrMo} 4-5$ constructional steel are presented. In the investigation step-cooling test treated samples were used. The step-cooling test process is a type of heat treatment simulating effects of passing time and environmental conditions (temperature and stress) on the sample. In the paper the method of testing the influence of stresses on the magnetic characteristics is presented. Frame shaped samples ensured a closed magnetic circuit and homogeneous distribution of stress. It was found that step-cooling test does not significantly influence the magnetoelastic characteristics and the structure of the samples. On the other hand, then tensile stresses significantly change the magnetic characteristics of $13 \mathrm{CrMo} 4-5$ constructional steel. That confirms the possibility of using measurements based on the magnetoelastic effect in the stress assessment for industrial non destructing testing of steel constructions.
\end{abstract}

DOI: 10.12693/APhysPolA.127.614

PACS: 46.25.Hf, 07.55.- $\mathrm{w}, 75.30 .-\mathrm{m}$

\section{Introduction}

Magnetoelastic characteristics of ferromagnetic materials are investigated from the time of discovery of this effect [1-3]. There are many known applications of this phenomenon, such as magnetoelastic force sensors $[4,5]$, magnetostrictive motors [6]. Recently, there are ongoing research into novel non-destructive testing (NDT) method development. In order to develop efficient methods of NDT, first the magnetoelastic characteristics of chosen constructional steels, related to stress [7], temperature [8] and material fatigue must be investigated. In the investigation heat resistant $13 \mathrm{CrMo} 4-5$ constructional steel was used. This steel is commonly used in energetic industry, for example as a material for overheated steam pipelines. Material fatigue can be simulated by the step-cooling test.

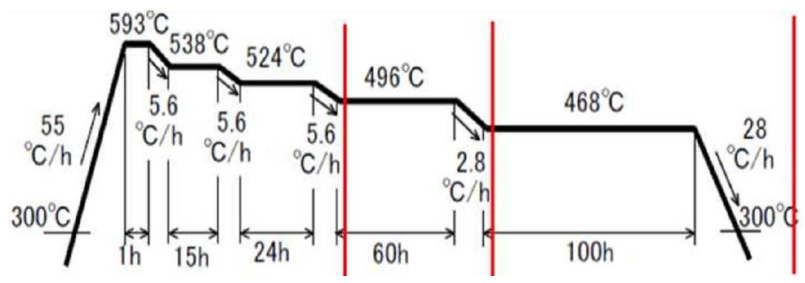

Fig. 1. Schematic of the step-cooling test.

${ }^{*}$ corresponding author; e-mail: d.jackiewicz@mchtr.pw.edu.pl
Step-cooling test is a heat treatment which affects the workpiece material in a manner of simulated influence of time and environmental conditions (temperature and stress). This process applies in particular to the accelerated heat treatment (couple hundred hours), allowing for the changes of the impact strength of the material evaluation in real conditions during long term (in the 100,000 $\mathrm{h}$ range) operation. In addition to the classical process of step-cooling test (Fig. 1), two other processes based on the classic treatment conditions were also utilized. In the first one (sample number 1) the treatment is interrupted after reaching the temperature of $524{ }^{\circ} \mathrm{C}$ and sample was annealed for $24 \mathrm{~h}$ at this temperature, while in the second (sample number 2) treatment was discontinued after reaching the temperature of $496^{\circ} \mathrm{C}$ and sample was annealed by $60 \mathrm{~h}$ at this temperature (Fig. 1). For each of the obtained test samples magnetoelastic properties investigation was performed. This procedure allowed the determination of characteristics for three different structures obtained by heat treatment simulating the degradation conditions in industrial environments. It should be noted, however, that the procedure used to modify the structure does not allow for the clear identification of the time after which given structure has a chance to emerge in the real operating conditions.

\section{Methodology of measurements}

For the investigation frame-shaped samples were used (Fig. 2). Such a shape enabled sample mounting for the test. Furthermore, the use of the window shaped sample allowed for the uniform distribution of the tensile stresses in the material and a closed magnetic circuit. 


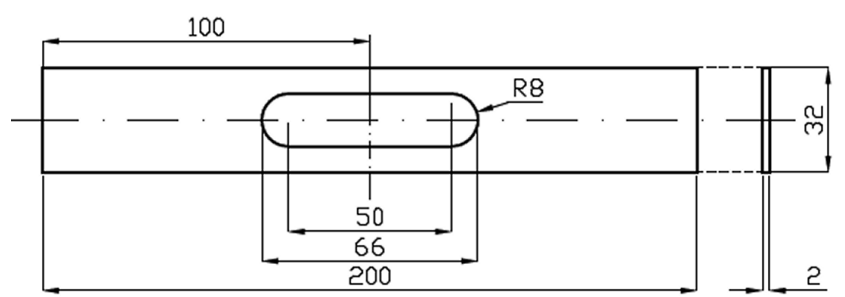

Fig. 2. Drawing of the frame-shaped sample.

Hysteresis loops measurements are done on a hysteresis graph HBL30PL. The oil hydraulic press is used for stress generating. For applying tensile stresses in the investigated sample, there is a special force reversing equipment. The reversor is turning the compressing force into tensile force, acting on the sample. For precise force measurements there is a force sensor, mounted in the axis of the reversor.

The sample was mounted in the reversor, and was investigated for magnetic properties as a function of applied stresses, in short for magnetoelasticity. The range of the applied stresses was from $0 \mathrm{MPa}$ to the rupture of the samples, at about $480 \mathrm{MPa}$. For obtaining the same initial conditions, before each of the measurements, the sample was demagnetized. Measurement series were done for a set value of the tensile stress, in each of the designated magnetizing field points. Next, the applied tensile stress was changed, and another measurement series was done. Tensile stresses were changed in the increasing way.

The subjects of the investigation were samples made of $13 \mathrm{CrMo} 4-5$ constructional steel after step-cooling test.

\section{Results}

Figure 3 presents the influence of tensile stresses $\sigma$ on the shape of $B(H)$ hysteresis loops of tree frame-shaped samples made of $13 \mathrm{CrMo} 4-5$ constructional steel. Shape of the magnetic hysteresis loop changes significantly under the tensile stresses $\sigma$ up to $450 \mathrm{MPa}$. For magnetizing field with $H_{\mathrm{m}}=850 \mathrm{~A} / \mathrm{m}$ amplitude, the flux density $B$ under such stresses decreases by about $53 \%$.

Figure 4 presents the magnetoelastic $B(\sigma) H_{\mathrm{m}}$ characteristics. On these characteristics the maximum field density $B$ may be observed. This maximum is called the Villari reversal point. Under the tensile stresses value of flux density $B$ in the samples first increases, and then, after reaching the Villari point it starts to decrease. Moreover, these changes are more pronounced for lower values of amplitude of magnetizing field $H_{\mathrm{m}}$. This occurs due to the fact that for lower values of magnetizing field $H_{\mathrm{m}}$, participation of magnetoelastic energy in the total free energy is significantly higher. It should be stressed that $B(\sigma) H_{\mathrm{m}}$ characteristics may be treated as a standard of presentation of magnetoelastic properties of constructional steels. Such standard could be especially useful from the point of view of mechanical stress assessment during non-destructive testing of construction elements.

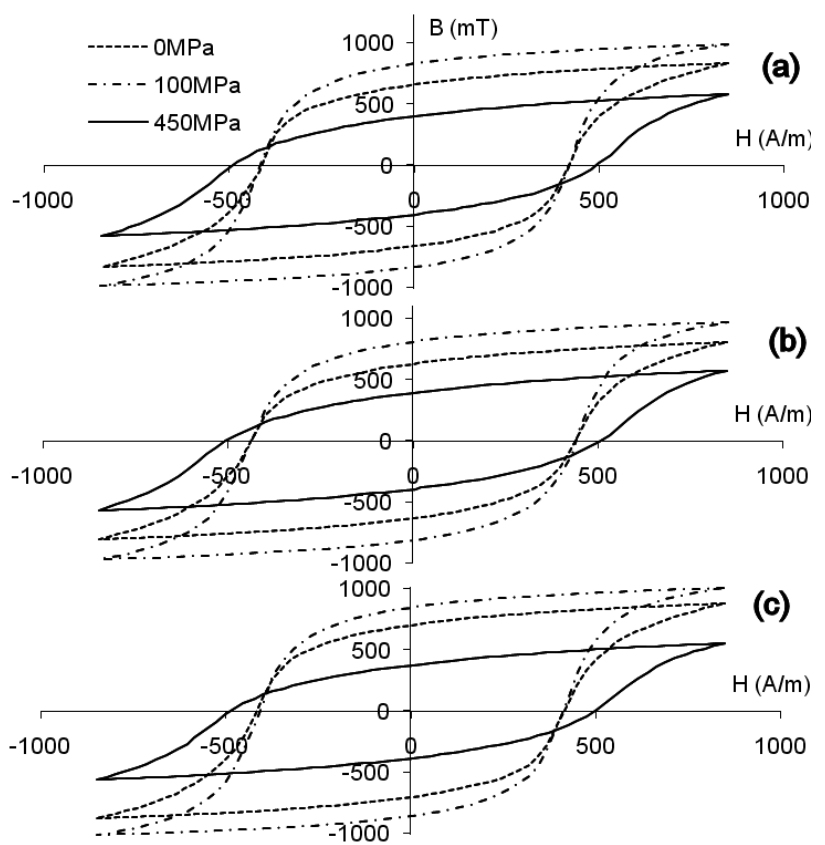

Fig. 3. The tensile stresses dependence of magnetic $B(H)$ characteristics of frame-shaped samples made of $13 \mathrm{CrMo} 4-5$ constructional steel after stepcooling test, for the amplitude of magnetizing field $\mathrm{H}_{\mathrm{m}}=850 \mathrm{~A} / \mathrm{m}$ : (a) sample 1, (b) sample 2, (c) sample 3.

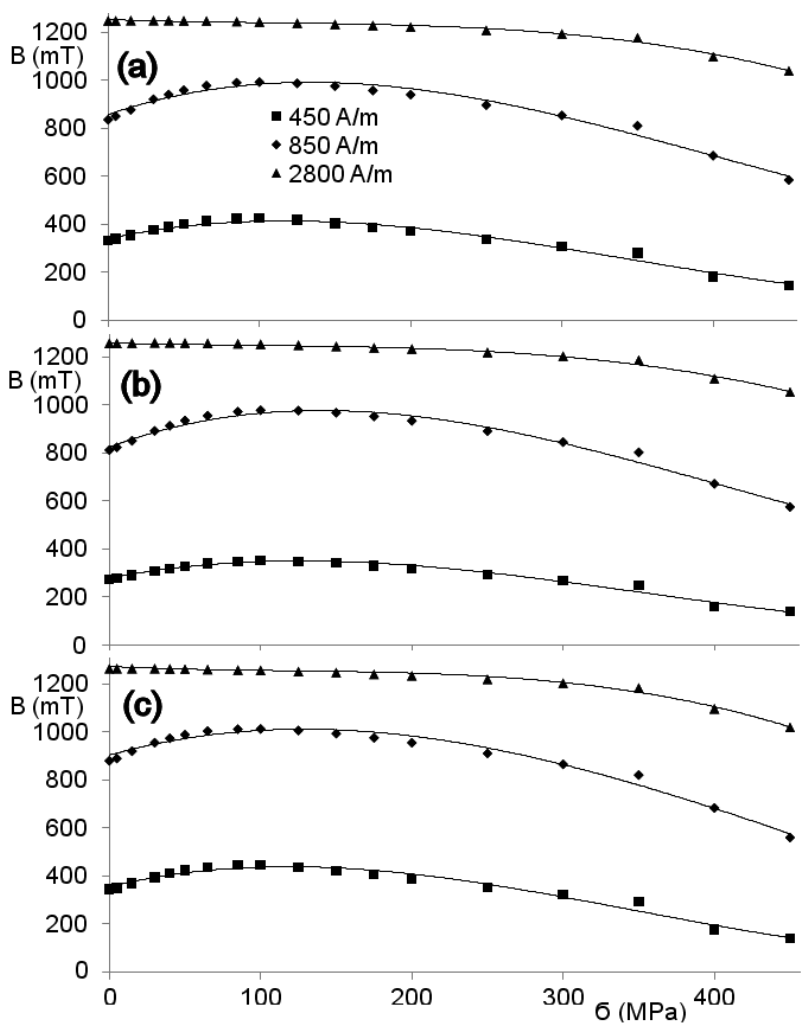

Fig. 4. The tensile stresses $\sigma$ dependences of flux density $B$ in 13 CrMo4-5 constructional steel after stepcooling test: (a) sample 1, (b) sample 2, (c) sample 3. 

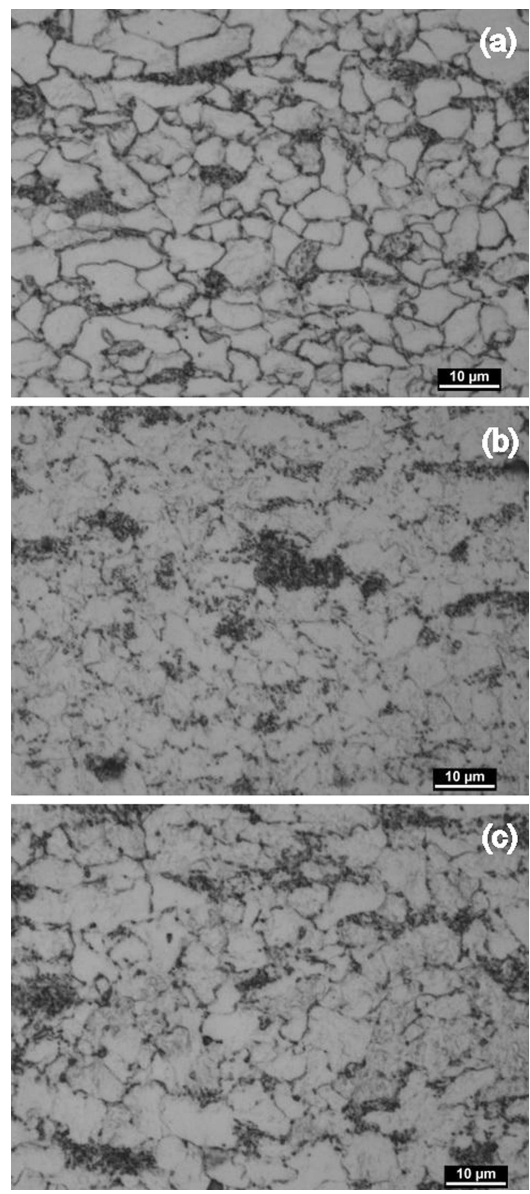

Fig. 5. Ferrite-carbide structure observed in the samples after the step-cooling test (also in broken variants, before the endof the process) (light microscopy, bright field, etched by Nital reagent 5\%): (a) sample 1, (b) sample 2, (c) sample 3.

It should be emphasized that the impact of the stepcooling test on magnetoelastic characteristics is not clear. For the three investigated frame-shaped samples made of $13 \mathrm{CrMo} 4-5$ constructional steel, the results obtained are similar.

The explanation for the above mentioned effect can be the lack of differences between the structures obtained in the three analyzed variants of the process. In all instances there was ferrite-carbide structure observed, with clearly visible grain of primary pearlite, in which there was an effect of lamellar structure decomposition (Fig. 5).

\section{Conclusions}

The test method presented in this paper opens up new possibilities for the description of the constructional steels magnetoelastic properties. Developed test stand allows us to determine these characteristics.

The experimental results of magnetoelastic properties of constructional steels confirm the validity of the proposed research methodology. Under the influence of tensile stresses, for low amplitude of the magnetizing field, the flux density changes significantly.

Changes of the magnetic characteristics are hardly influenced by temperature. From the results it can be concluded that the $13 \mathrm{CrMo} 4-5$ steel is temperature resistant. This allows the use of magnetoelastic characteristics for non-destructive testing of constructional steel structures, because they are not influenced by temperature.

\section{Acknowledgments}

This work was partially supported by the National Centre of Research and Development (Poland) within grant no. PBS1/B4/6/2012.

\section{References}

[1] R. Bozorth, Ferromagnetism, Van Nostrand, New York 1951

[2] S. Chikazumi, Physics of Ferromagnetism, Clarendon Press, Oxford 1997.

[3] K. Perduta, J. Olszewski, S. Busbridge, M. Nabialek, Acta Phys. Pol. A 114, 1537 (2008).

[4] J. Seekircher, B. Hoffmann, Sensors Actuat. A Phys. 22, 401 (1989).

[5] A. Bienkowski, R. Szewczyk, J. Salach, Acta Phys. Pol. A 118, 1008 (2010).

[6] J.S. Park, O.K. Oh, Y.W. Park, N.M. Wereley, IEEE Trans. Magn. 49, 3379 (2013).

[7] D. Jackiewicz, R. Szewczyk, J. Salach, A. Bienkowski, K. Wolski, in: Recent Technological and Scientific Advances: Mechatronics 2013, Eds. J. Ryszard, B. Tomas, Springer 2014, p. 479.

[8] D. Jackiewicz, R. Szewczyk, J. Salach, Solid State Phenom. 199, 466 (2013). 